\title{
Signaling through RIG-I and type I interferon receptor: Immune activation by Newcastle disease virus in man versus immune evasion by Ebola virus (Review)
}

\author{
VOLKER SCHIRRMACHER \\ Division of Tumorimmunology, The Immunological and Oncological Center of Cologne, D-50674 Cologne, Germany
}

Received March 4, 2015; Accepted April 24, 2015

DOI: $10.3892 /$ ijmm.2015.2213

\begin{abstract}
In this review, two types of RNA viruses are compared with regard to the type I interferon (IFN) response in order to obtain a better understanding of the molecular mechanisms of immune activation or evasion. Upon human infection, both viruses exert either beneficial or detrimental effects. The Newcastle disease virus (NDV), is a type strain for avian paramyxoviruses, while the Ebola virus (EBOV), is a virus affecting primates. During evolution, both viruses specifically adapted to their respective hosts, acquiring sophisticated viral escape mechanisms. Two types of receptors play an important role in the life cycle of these two viruses: cytoplasmic retinoic acid-inducible gene I (RIG-I) and membrane expressed type I IFN receptor (IFNAR). In mouse and human cells, NDV is a strong inducer of the type IIFN response. The early phase of this is initiated by signaling through RIG-I and the late response by signaling through IFNAR. EBOV does not induce type I IFN responses in humans as it has viral proteins that specifically and strongly interfere with RIG-I and IFNAR signaling, as well as immune activation. In this review, we discuss whether the beneficial effects of one virus can be exploited in the fight against the detrimental effects of the other.
\end{abstract}

\section{Contents}

1. Introduction

2. Newcastle disease virus

3. Ebola virus

4. Conclusions

5. Prospects

Correspondence to: Professor Volker Schirrmacher, Division of Tumorimmunology, The Immunological and Oncological Center of Cologne, Hohenstaufenring 30-32, D-50674 Cologne, Germany

E-mail: v.schirrmacher@web.de

Key words: interferon receptors, interferons and cytokines, infectious disease, viral evasion/potentiation, signal transduction

\section{Introduction}

Interferons (IFNs) were first characterized for their ability to 'interfere' with viral replication, and indeed one of their major functions is the establishment of a robust antiviral state in response to infection $(1,2)$. In addition, IFNs activate immune cells and facilitate the recognition of virus-infected cells and tumor cells by the immune system, as they stimulate antigen presentation to T lymphocytes (3). IFNs are usually subdivided into three classes: type I (including IFN- $\alpha$, IFN- $\beta$ and IFN- $\omega$ ), type II (in humans, IFN- $\gamma$ ) and type III [including interleukin (IL)-28 and IL-29] (3). Type I IFNs are produced by virus-infected cells. Plasmacytoid dendritic cells (DCs) and mononuclear phagocytes are the major sources of IFN- $\alpha$ (4), while IFN- $\beta$ is produced by a number of cell types, including fibroblasts (5).

IFN- $\beta$ is the principal antiviral factor secreted by infected mammalian cells in response to the activation of retinoic acid-inducible gene (RIG-I) following Newcastle disease virus (NDV) infection. Type I IFNs bind to the IFN- $\alpha$ membrane receptor (IFNAR) (2) on infected cells and through Janus kinase (JAK) and signal transducer and activator of transcription (STAT) signaling (6), induce the expression of genes whose products enhance the susceptibility of cells to cytotoxic natural killer (NK) cell- and T cell-mediated killing (7). In addition, type I IFNs induce resistance to viral replication in all cells, thereby involving autocrine and paracrine actions (7).

In this review, immune activation by NDV in humans is compared to immune evasion by Ebola virus (EBOV). Such timely comparison is justified as both phenomena are associated with the activation of the same two signaling pathways.

Unlike NDV, which is a pathogen found in birds but not in humans, EBOV, as a virus affecting primates, is a devastating pathogen affecting humans. During approximately 200 million years of evolution, viruses from mammals, (derived from therapsids), have had time to adapt to the immune systems of their host. According to a recent whole-genome analysis, 95\% of bird species (derived from sauropsids) developed during a rapid radiation following the Cretaceous-Paleogene mass extinction approximately 66 million years ago (8). Thus, bird viruses have had a shorter time period for adaptation to the host immune system than mammalian viruses. The characteristics of the two RNA viruses, NDV and EBOV, are reviewed herein with particular focus on the aspect of the 
species-specificity of viral escape mechanisms antagonizing type I IFN responses.

\section{Newcastle disease virus}

NDV in birds. NDV is one of the most important diseases affecting poultry worldwide. NDV outbreaks were first reported in Indonesia, and, subsequently, in Newcastle-upon-Tyne in the year 1926. Infections by virulent NDV strains cause severe economic losses and may have flock mortality rates of up to $100 \%$. Therefore, NDV has a significant impact on the world economy, possibly more so than any other disease affecting animals (9).

NDV belongs to the avian paramyxovirus serotype (APMV)-1 family and is the most characterized member among the nine APMV serotypes. It is possible that all species of birds are susceptible to NDV infection. However, the disease may vary greatly depending upon the virus strain and the host species. Eighteen NDV strains from four lineages have been identified and classified as velogenic, mesogenic and lentogenic according to their pathotypes (10). NDV attaches to respiratory epithelial cells through the viral HN protein, which binds to sialic acid containing cell surface receptors, such as gangliosides and $\mathrm{N}$-glycoproteins. This is followed by the activation of the F protein, which leads to the fusion of the viral and the host cell membranes. In the cytoplasm of the host cell, the viral genome, a $15 \mathrm{~kb}$ non-segmented negative single-stranded RNA (ssRNA), is transcribed into mRNAs and is translated into viral proteins (11). Respiratory disease can be mild in the case of lentogenic viruses, more severe with mesogenic viruses and severe with a high mortality rate in the ase of velogenic viruses. Velogenic viruses are further subdivided into viscerotropic, which cause mortality with haemorrhagic lesions in the intestines, and neurotropic, when neurological diseases predominate without haemorrhagic lesions in the intestines (12).

All NDV strains encode seven proteins: N, P, V, M, F, $\mathrm{HN}$ and $\mathrm{L}$. The $\mathrm{V}$ protein is not essential for viral replication in vitro and serves as an accessory protein. The $\mathrm{V}$ protein is a frameshift variant of the NDV phosphoprotein P. and, while the $\mathrm{P}$ protein consists of 395 amino acids, the $\mathrm{V}$ protein consists of only 239 amino acids. The incorporation of two $\mathrm{G}$ nucleotides at the RNA editing site of the $\mathrm{P}$ protein results in the frameshift variant protein $\mathrm{V}$ (13). The $\mathrm{V}$ protein of NDV has been shown to inhibit the IFN response in birds in two ways: i) through the inhibition of IFN signaling by targeting STAT1 for degradation (14); and ii) through interaction with melanoma differentiation-associated gene 5 (MDA5), leading to the inhibition of interferon regulatory factor 3 (IRF-3) activation and IFN- $\beta$ induction (15).

Of note, it has been demonstrated that the $\mathrm{V}$ protein of NDV is a determinant of host range restriction. Recombinant NDV (rNDV) mutants, which are defective in the expression of $\mathrm{V}$ protein, grow poorly in embryonated chicken eggs and chicken embryo fibroblasts compared to wild-type (WT) rNDV. Furthermore, the NDV V protein has been shown to play an important role in preventing apoptosis in a species-specific manner. It has been suggested that the host range of NDV is limited by the specificity of its $\mathrm{V}$ protein for bird proteins to efficiently prevent innate host defenses, such as the IFN response and apoptosis (16-18).
In recent years, NDV has drawn a lot of research interest, not only due to the fact that it is an important pathogen affecting poultry, but also that in man, it exerts fascinating oncolytic and immune stimulatory effects. It also has potential for use as a novel vaccine vector for the treatment of diseases in humans and animals (10).

\section{NDV in mouse and man}

The NDV-induced type I IFN response. Upon the infection of mouse or human cells with the NDV bird virus, an uninhibited type I IFN response (19) is initiated, as the viral V protein cannot interact with the proteins from mammalian cells. It is of particular significance that the activation of a rapid and strong type I IFN response by NDV in normal mouse or human cells prevents viral replication, cytotoxic effects and disease pathology in normal tissues. Thus, NDV is not considered a pathogen in mouse or man and shows a high safety profile as regards its clinical application.

A diagram of the cellular response in mouse and man is illustrated in Fig. 1. It shows an early- and a late-phase response. During the early phase, the antiviral response of normal (non-tumor) cells is initiated through the recognition of viral RNA by two types of pathogen recognition receptors: i) endosomal Toll-like receptors (TLRs), particularly TLR3 and ii) cytoplasmic RIG-I-like receptors (RLRs). RIG-I has been shown to be the cytoplasmic viral RNA receptor for NDV $(20,21)$. Of note, RIG-I binds specifically to RNA containing 5'-phosphate, such as viral RNA, while mammalian RNA is either capped or contains base modifications (22). Once activated, RIG-I binds to the adaptor protein IFN- $\beta$ promoter stimulator-1 (IPS-1) which, after a further signaling cascade, activates, IRF-3 during the early phase. This transcription factor (TF) is then phosphorylated, translocates to the nucleus and induces the IFN response (23). IRF-3 plays an important role in the IFN response of mouse macrophages to NDV infection (24).

RIG-I triggering does not only involve the upregulation of RNA copies. RIG has the structural combination of an $\mathrm{N}$-terminal caspase recruitment domain (CARD) not and a C-terminal RNA helicase domain with which it interacts with the viral non-capped RNA (25). Following the recognition of small viral RNAs, RIG-I elicits signaling cascades, which eventually lead to the activation of the nuclear factor (NF)- $\kappa B$ and IRF-3 TFs. NF- $\kappa$ B regulates the production of most cytokines and chemokines (26), while IRF-3 is central to the development of an antiviral state through the induction of antiviral genes (27). The rapid and robust expression of type I IFN genes is a prerequisite for the induction of numerous antiviral proteins that modulate protein synthesis, growth arrest and apoptosis (28).

During the late phase of the IFN response, the type I IFN molecules secreted during the early phase interact with the cell surface, express IFNAR and initiate an amplification loop of the IFN response, which involves STAT proteins and IRF-7 (29). IFNAR (2) is expressed by virtually all cells in the body and consists of IFNAR1 and IFNAR2 chains. The cytoplasmic domains of IFNAR1 and IFNAR2 are physically associated with the JAKs, Tyk2 and JAK1, respectively. Ligands binding IFNAR represent a large family of four-helix bundle cytokines, numbering close to 20 in humans and mice. Upon ligand 


\section{Early phase}

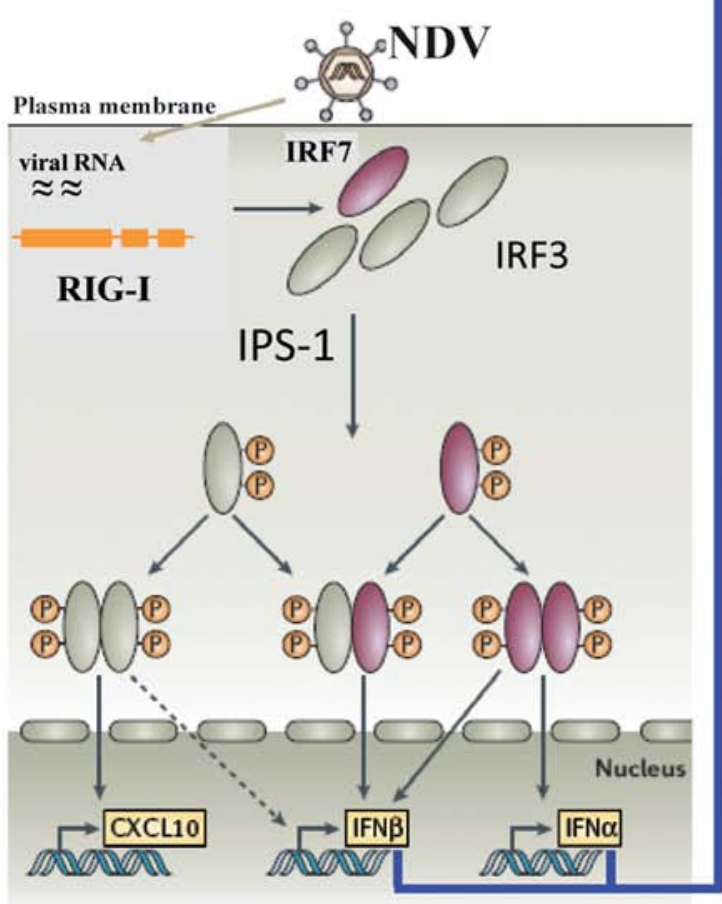

\section{Late phase}

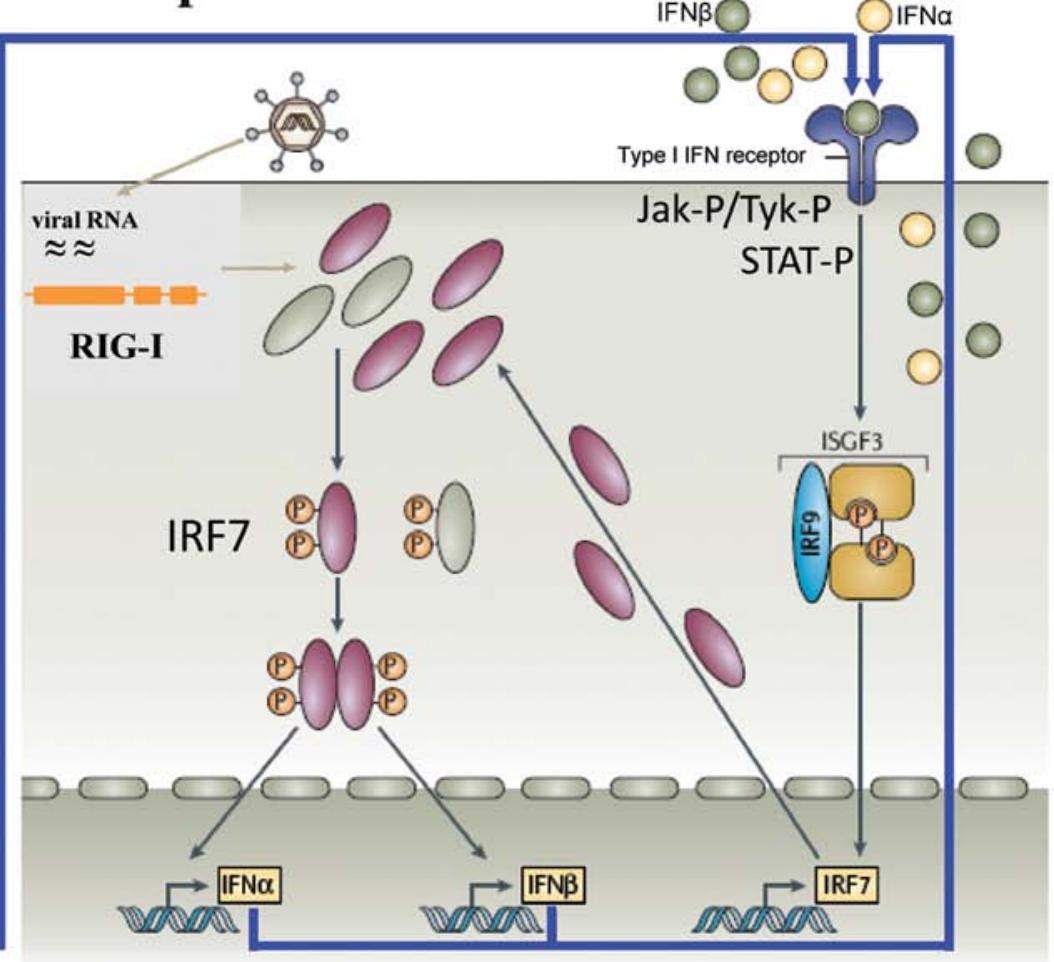

Figure 1. Diagram of the uninhibited Newcastle disease virus (NDV)-induced type I interferon (IFN) response in mouse and human cells. Following cell membrane attachment and the fusion of viral and host cell membranes, the viral RNA enters the cytoplasm where it is immediately recognized as foreign by retinoic acid-inducible gene I (RIG-I). This initiates a signaling cascade involving the transcription factors (TFs), IRF-3 and IRF-7. Following phosphorylation and dimerization, these protein complexes enter the nucleus and initiate the transcription of genes coding for chemokines (CXCL10 and IP-10) for effector T cell recruitment or for type I IFNs (IFN- $\beta$ and IFN- $\alpha$ ). The average upregulation time for these genes is $8-12 \mathrm{~h}$. This time period corresponds to the early phase of the response. The late phase is initiated following interaction of the released type I IFNs as ligands with the corresponding type I IFN membrane receptor. A feedback loop signal activation cascade involving further protein complexes, such as ISGF3, then leads to response amplification and the induction of further antiviral response genes.

binding, Tyk 2 and JAK 1 become activated by transphosphorylation (6). These, in turn, phosphorylate receptor tyrosine(s), directing the recruitment of inactive STAT1 and STAT2. At the receptor, STAT1 and STAT2 are activated by phosphorylation $(29,30)$. The STAT proteins then heterodimerize and with the IFN regulatory factor IRF-9, to form a complex known as ISGF3 (Fig. 1). This translocates to the nucleus to bind to the IFN-stimulated response element (ISRE). This DNA-binding complex directs the expression of IFN-stimulated genes (ISGs) that create the antiviral state in the target cells and block viral replication.

Importance of the IFN receptor-mediated feedback amplification loop. IFNAR plays a crucial role in the IFN feedback amplification loop and its consequences. This is illustrated in Fig. 2, which shows the results of the NDV infection of murine DCs from either C57BL/6 WT mice or IFNAR gene knockout (KO) mice. Fig. 2A (panel a) shows viral replication $10.5 \mathrm{~h}$ following infection by NDV (strain Ulster) assessed by RT-PCR of the viral M gene. Over 3,000 M gene copies were obtained from the DCs of $\mathrm{KO}$ mice in comparison to $<100$ copies from cells obtained from WT mice. Cell culture supernatants were tested for the content of the cytokines, Il-12, IFN- $\alpha$ and tumor necrosis factor (TNF)- $\alpha$. The NDV infection of DCs from WT mice induced a strong expression of genes coding for IL-12p70 (Fig. 2A, panel b), IFN- $\alpha$ and TNF- $\alpha$ (Fig. 2 C, panels a and b), while this was not the case with DCs from $\mathrm{KO}$ mice.

In DCs from WT mice, the produced IFN- $\alpha$ caused a feedback loop stimulation through IFNAR. This resulted in the suppression of viral replication and the expression of pro-inflammatory cytokines, such as TNF- $\alpha$ and IL-12. In the cells from KO mice, neither RIG-I nor IRF-7 were upregulated upon NDV infection, in contrast to the cells from WT mice (Fig. 2B, panels a and c). Further results with DCs from mice deficient in either IRF-3 or IRF-7, or from IRF-3/-7 double $\mathrm{KO}$ mice revealed that RIG-I triggering by NDV replication in mouse DCs induced IL-12 production independently of the IRF-3 and IRF-7 pathway (31).

DCs function to maintain tissue-specific tolerance or they can be immunogenic and initiate antigen-specific $\mathrm{T}$ cell immunity. It depends on the microenvironment in vivo and on inflammatory stimuli whether immature DCs with functional plasticity differentiate into tolerogenic or immunogenic DCs with stable phenotypes. Murine DCs, upon infection with $\mathrm{NDV}$, differentiate into the immunogenic phenotype DC1 characterized by the secretion of pro-inflammatory cytokines, in particular IL-12 and IFN- $\alpha / \beta$ (31). The priming or 
A
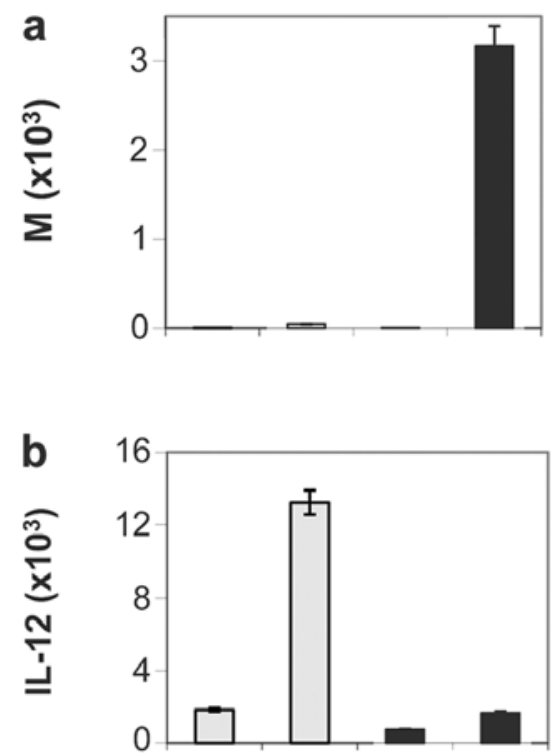

NDV
B

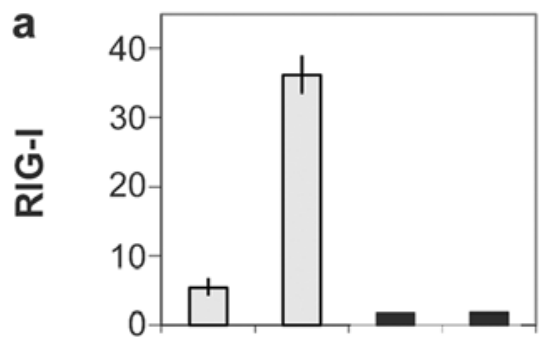

b

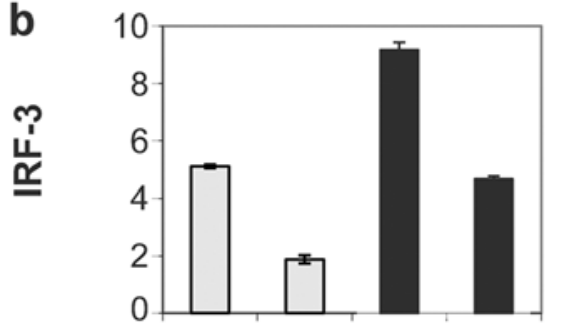

C

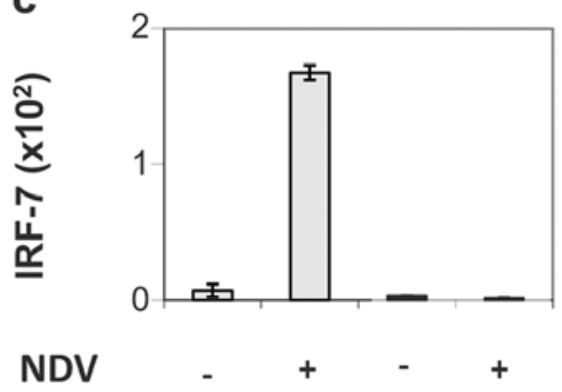

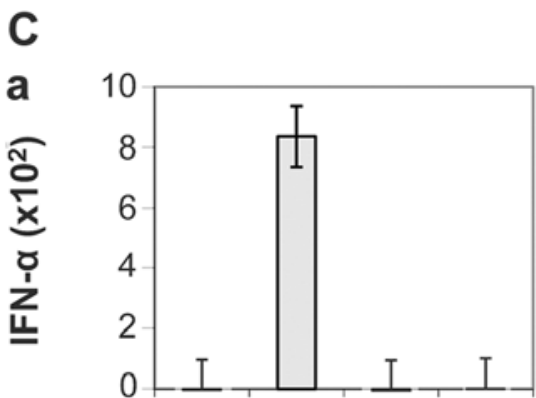

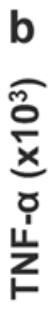

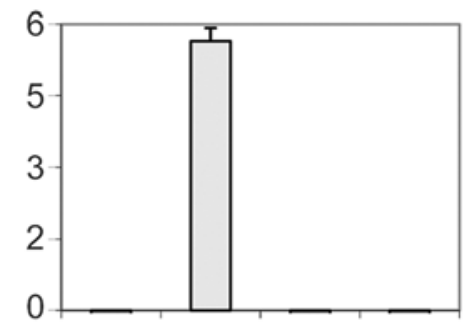

NDV

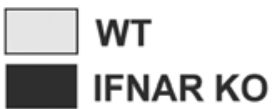

Figure 2. Comparison of murine dendritic cells (DCs) from wild-type (WT) and type I IFN receptor (IFNAR) knockout (KO) mice in response to infection by Newcastle disease virus (NDV). DCs from C57BL/6 WT or IFNAR1 KO mice were generated from bone marrow-derived mononuclear cells, plated in 24-well plates $\left(5 \times 10^{5} /\right.$ well) and infected with NDV (lentogenic strain Ulster, 30 hemagglutinating units per $10^{6}$ cells) for $10.5 \mathrm{~h}$. Viral replication was assessed by RT-PCR of the viral M gene. This was quantified from cDNA of isolated RNA using a fluorescent oligonucleotide probe system. RT-PCR was also used to detect the genes retinoic acid inducible gene I (RIG-I), IRF-3 and IRF-7. The concentration of murine cytokines IL-12p70 and TNF- $\alpha$ in supernatants was determined by a Cytometric Bead Array and analyzed on the FACSArray (both from BD Biosciences, Heidelberg, Germany). Mouse type I interferon (IFN)- $\alpha$ in supernatants of cultured cells was determined by ELISA using a rat monoclonal antibody and a rabbit polyclonal antibody against murine IFN- $\alpha$ (both from PBL Assay Science, Piscataway, NJ, USA) (32). All cytokine protein concentrations are given in $\mathrm{pg} / \mathrm{ml}$. (A) Viral replication associated M gene expression (a) and production of IL-12p70 (b); (B) expression of RIG-I (a), IRF-3 (b) and IRF-7 (c); (C) production of IFN- $\alpha$ (a) and TNF- $\alpha$ (b).

programming for DC1 involves two receptor-initiated signaling cascades, the first one initiated through cytoplasmic RIG-I, and the second through membrane-expressed IFNAR $(21,32)$.

NDV, a prototype avian virus that may be used to study an uninhibited cellular response to viral infection in human $D C s$. We investigated the effects of NDV on human DCs by analyzing the release of cytokines important for Th1 or Th2 polarization. Human monocyte-derived DCs were found to become polarized towards DC1 by in vitro stimulation with NDV (33).

Pathogenic viruses subvert normal immune functions in DCs through the expression of immune antagonists $(18,34)$. Understanding how these antagonists interact with the host immune response requires knowledge of the underlying genetic regulatory network that operates during an uninhibited antiviral response. Such a network was identified by studying human DCs and their response to infection by NDV (19), knowing that this virus is able to stimulate innate immunity and DC maturation through the activation of RIG-I signaling and lacks the ability to evade the human IFN response.

A new approach was developed, integrating genome-wide expression kinetics and time-dependent promoter analysis. It was found that the genetic program underlying the antiviral cell-state transition during the first $18 \mathrm{~h}$ post-infection can be explained by a single convergent regulatory network. Gene expression changes were driven by a step-wise multi-factor cascading control mechanism, where the specific TFs controlling expression changed over time. This study of systems biology involved, among others, microarray experiments, microarray analysis, transcription factor binding site analysis, time-dependent promoter analysis, electromobility shift assay and regulatory network construction (19). Through all these new tools, this analysis revealed a robust antiviral transcriptional network that may be induced in human DCs by infection with NDV. Table I lists the 24 critical TFs and their time of expression following infection with NDV. The timing of this program appeared as highly conserved. 
Table I. NDV infection of human DCs: Kinetics of upregulated transcription factors.

\begin{tabular}{lclc}
\hline $\begin{array}{l}\text { Transcription } \\
\text { factor }\end{array}$ & $\begin{array}{c}\text { Hours } \\
\text { p.i. }\end{array}$ & $\begin{array}{c}\text { Transcription } \\
\text { factor }\end{array}$ & $\begin{array}{c}\text { Hours } \\
\text { p.i }\end{array}$ \\
\hline IRF-1 & 2.5 & IRF-7 & 3 \\
STAT1 & 4 & STAT2 & 4 \\
ATF3 & 5 & IRF-2 & 6 \\
CREM & 6 & MAX & $7-8$ \\
STAT3 & $7-8$ & RUNX3 & 8.5 \\
RELA & 8.5 & FOXC1 & 8.5 \\
IRF-8 & $9-10$ & ALX1 & $9-10$ \\
TGIF1 & $10-11$ & IRF & $11-12$ \\
STAT4 & $11-12$ & NFB & 12 \\
NF- 6 2 & 12 & EGR4 & 12.5 \\
FOX03 & 13.5 & ZEB1 & 14 \\
REL & 14.5 & STAT5A & 18 \\
\hline
\end{tabular}

The transcription factors were placed in the time slice during which the gene was first differentially expressed. The 24 transcription factors build a regulatory network. Regulatory relationships can be either feed-forward, feedback or reciprocal. This network induces 779 of 1,351 (58\%) upregulated cellular genes post infection (p.i.) of dendritic cells (DCs) with Newcastle disease virus (NDV) (24).

The described network of TFs spans virtually the entire time-period analyzed. Of the $24 \mathrm{TFs}, 18$ appear in the known general pathogen response signature (35) or in the core DC response signature (36). The TFs are predicted to regulate 779 of the 1,351 upregulated genes. The network contains both feed-forward links, which propagate the transcriptional signal through time, as well as feedback links, where TFs may influence the activity of targets that have previously been upregulated. It was concluded that the proposed network is effective in capturing the underlying biology and produces a pattern that is consistent with a step-wise transcriptional signal propagation.

Tumor-selective replication, safety and the activation of immune cells. Integral to the life cycle of all RNA viruses is the formation of double-stranded RNA (dsRNA), which activates a spectrum of cellular defence mechanisms involving IFN- $\alpha / \beta$. Tumors from mouse or man provide a relatively permissive substrate for the propagation of RNA viruses, such as NDV as mutations in tumor cells often cripple the IFN system to allow uninhibited proliferation and to provide resistance to apoptosis (37). The first step of infection with NDV takes place in all cell types of mouse or man, whereas the second step (which corresponds to viral replication) occurs only in tumor cells since it is stopped rapidly in normal cells. The replication of NDV involves the use of the full-length viral antigenome as a template. This step is prevented in non-tumorigenic mouse or human cells (37).

An inverse correlation was found between the expression of four antiviral genes and the susceptibility of cells to infection with NDV: i) RIG-I, ii) IRF-3, iii) IFN- $\beta$ and iv) IRF-7 (20). In addition, the membrane receptor IFNAR was demonstrated to be of great importance (21). The basic or induced levels of the four aforementioned genes were higher in the normal cells than in the tumor cells. In addition, signaling through IFNAR was often found to not be fully functional in tumor cells. Taken together, these observations explain the high safety profile of NDV upon human application $(10,11)$.

The activation of immune cells is a further factor for the safety of this virus. In vitro infection with NDV has been shown to cause the activation of human NK cells (38), human monocytes (39) and had a co-stimulatory effect on CD4 (40) and CD8 (41) mouse and human T cells. The activated cells exerted cytotoxic effects through NKp46 (38), TNF-related apoptosis-inducing ligand (TRAIL) (39) and released nitric oxide (NO) (42) and pro-inflammatory cytokines (31).

\section{Ebola virus}

$E B O V$ in man. The Filoviridae family of viruses includes the genera EBOV and Marburg virus (43). EBOV was first discovered in 1976. With a diameter of $80 \mathrm{~nm}$ and a length of up to $14,000 \mathrm{~nm}$, EBOV belongs to the largest known RNA viruses. Similar to NDV, the genome consists of a negative ssRNA. It codes for eight proteins, two of which are the viral proteins, VP24 and VP35, which are discussed below.

EBOV infects primates (gorillas, chimpanzees and humans). The primary target cells are macrophages and DCs (44). The zoonotic transmission of EBOV to humans causes severe and often times lethal hemorrhagic fever. The disease characteristics are systemic inflammatory response syndrome (SIRS), disseminated intravascular coagulation (DIC), systemic hemorrhage and multiple organ failure (45). EBOV shuts down the host's innate and adaptive immune systems. It then replicates uncontrollably and causes a cytokine storm in the host (46).

Filoviral infections in primates are associated with ineffective innate antiviral responses as a result of virally encoded immune antagonists. These render the host incapable of mounting effective innate or adaptive immune responses. During the first 3 weeks after infection, the release of endogenous pyrogenes (IL-1 $\beta$, IL- 6 and TNF- $\alpha$ ) is prevented. Several filoviral encoded components target type I IFN responses. Many of these innate immune suppression mechanisms that are important for viral replication and pathogenesis are species-specific (47).

EBOV: a virus-inhibited IFN response. Two of the eight viral proteins of EBOV are involved in immunosuppression $(48,49)$. They prevent type I IFN signaling in multiple ways, which is also the topic of the present review. While VP35 antagonizes the early phase of the IFN response, VP24 is an antagonist of the late phase. The EBOV VP35 protein binds directly to dsRNA and inhibits several antiviral signaling pathways. It acts as a component of the viral RNA polymerase complex, a viral assembly factor and an inhibitor of host IFN production. Mutation of selected basic residues within the C-terminal half of VP35 abrogates its dsRNA-binding activity, impairs VP35-mediated IFN antagonism, and attenuates EBOV growth in vitro and in vivo. The structure of the C-terminal VP35 IFN inhibitory domain (IID), solved to a resolution of $1.4 \AA$, revealed a unique fold centered on Arg-312 (48). In an analogous study, Marburg virus VP35 proteins were demonstrated to 
be capable of fully coating the backbone and capping the ends of dsRNA for IFN antagonism (50). Furthermore, it was shown that conserved basic residues in the IID recognize the dsRNA backbone, whereas the dsRNA blunt ends are 'end-capped' by a pocket of hydrophobic residues that mimic the RLR recognition of blunt-end dsRNA, the initiation step of the early-phase response (Fig. 1, early phase) (51).

VP35 also blocks virus-induced IRF-3 phosphorylation, subsequent IRF-3 dimerization and nuclear translocation. VP35 thus inhibits the early induction of antiviral genes, including the IFN- $\beta$ gene (Fig. 1, early phase). VP35 is also capable of preventing the IRF-3-dependent activation of the IFN- $\alpha 4$ promoter in response to viral infection (52). It is also able to inhibit the antiviral response induced by IFN- $\alpha$ (Fig. 1, late phase). The phosphorylation of the dsRNA-dependent protein kinase (PKR) and of the elongation initiation factor eIF-2 $\alpha$ was also suppressed in cells expressing VP35 (53). A single amino acid change in the VP35 protein was demonstrated to be capable of reversing the inhibition of host innate immune responses. Thus, infection with a mutated recombinant virus (recEbo-VP35/R312A) resulted in a strong innate immune response, including the increased expression of MDA5, RIG-1, regulated on activation, normal $\mathrm{T}$ cell expressed and secreted (RANTES), monocyte chemoattractant protein-1 (MCP-1), ISG15, ISG54, ISG56, ISG60, STAT1, IFN- $\beta, 2,5$-oligoadenylate synthetase (OAS) and myxovirus (influenza virus) resistance 1 (MX1) (54).

During antiviral defence, IFNAR signaling triggers the nuclear transport of tyrosine-phosphorylated STAT1 (PY-STAT1), which occurs through a subset of karyopherin $\alpha$ (KPNA) nuclear transporters. EBOV VP24 (eVP24) binds directly to STAT1 and inhibits its nuclear translocation, a step of the late-phase feedback loop (Fig. 1). Recently, it has been demonstrated that eVP24 targets a unique nuclear localization signal (NLS) binding site on KPNA to selectively compete with nuclear import of PY-STAT1 (49). It leaves the transport of other cargo that may be required for viral replication unaffected. New crystal structures of VP24 derived from pathogenic and non-pathogenic EBOV revealed a pyramidal fold with sites required for virulence and for STAT1 binding (55). Such studies offer templates for drug design, and provide the three-dimensional framework necessary for the biological dissection of the many functions of VP24 in the virus life cycle.

\section{Conclusions}

The comparison of the two viruses, NDV and EBOV, in this review demonstrates that RNA viruses with a similar genome may exert completedly different effects in man. Depending on whether they are derived from birds or primates, they can exert either beneficial or detrimental effects. Signals delivered through RIG-I and IFNAR cause immune activation, which is the case with NDV, or they are antagonized causing immune evasion, which is the case with EBOV.

The infection of human DCs with NDV induces a robust uninhibited antiviral response which prevents viral replication, and differentiates and polarizes the cells towards DC1-activating Th1 cells. When NVD infects mouse or human cells it activates a multitude of genes, cells and cellular activities. In vivo, such viral 'priming' of cells may lead to immune system stimulation or immune system conditioning, which mostly relies on the effects of the induced type I IFN response.

Signaling through RIG-I and IFNAR may have far-reaching consequences for the antiviral immune response. The activation of the RIG-I pathway is able to reduce the antigen requirement by 10 - to 100 -fold in inducing optimal influenza-specific cellular and humoral responses, including protective immunity. These effects include an enhanced germinal center reaction, $\mathrm{T}$ follicular helper cell responses, antibody affinity maturation and plasma cell responses in draining lymph nodes, the spleen and bone marrow. These effects are dependent on type I IFN and IPS-1 signaling, but are independent of the MyD88- and TLR3-mediated pathways (56). Since type I IFN receptors are expressed by virtually every type of cell in the body, the ligand-induced IFN signaling cascades have far-reaching consequences. For instance, type I IFN-mediated crosstalk between plasmacytoid DCs on one side and macrophages and conventional DCs on the other, secure the control of fatal cytopathic mouse hepatitis virus (57).

Primate-derived EBOV succeeds in antagonizing the human IFN response by two proteins, VP35 and VP24, which specifically target signals through RIG-I and IFNAR, respectively, thereby demonstrating the importance of these coordinated signaling systems. The bird-derived virus, NDV, has only one protein, the frameshift variant $\mathrm{V}$ protein, with which to antagonize the IFN response. Perhaps this difference can be accounted for by the difference in the respective time periods available for adaptation during evolution. EBOV may be more potent than NDV in antagonizing the IFN response as it has two, instead of only one, inhibitory proteins. However, it is likely that it is not the quantity but the quality of inhibitory proteins that matters $(58)$.

\section{Prospects}

Due to its high safety profile in human application $(10,11)$, NDV may be employed not only as an oncolytic agent in cancer patients, but also as an agent for immune stimulation and for prophylacting conditioning of the host immune system against the risk of viral infection. This may be particularly relevant for individuals whow come into contact with patients infected with EBOV. Such immune-conditioning pre-treatment would 'prime' the cells and establish a state of increased viral resistance. In this way, one virus, the avian NDV, may exert beneficial effects against the other virus, EBOV. Beneficial effects can be expected in particular during the early non-symptomatic phase of infection. It has been suggested that applying a non-specific antiviral approach during the incubation period of viral infection is an essential protective approach which renders the host immune sytem into an alert state, thus attenuating viral replication (46). The use of an IFN-inducing agent, such as NDV, may be thus more effective and may cause less severe side-effects than the use of IFN- $\beta$, which has also been suggested (59).

Other immunological means for counteracting EBOV infection may be based on neutralizing antibodies, particularly when isolated from memory B cells (60) of patients who have survived the infection. In addition, libraries of memory $\mathrm{T}$ cells may be developed from such patients to screen the $\mathrm{T}$ cell immune repertoires for protective activity, function and specificity (61). This is supported by a recent immune analytical study (62). 
In connection with the EBOV epidemic in West Africa in 2014, a vaccine was developed by the Canadian National Microbiology Laboratory on the basis of recombinant vesicular stomatitis virus (rVSV) expressing the filovirus glycoprotein of the lethal Zaire ebolavirus (ZEBOV). Antibodies were reported as necessary for rVSV/ZEBOV-GP-mediated protection against lethal EBOV challenge in non-human primates (63). VSV, similar to NDV and EBOV, is a negative ssRNA virus (64). Similar to rabies, it belongs to the family of Rhabdoviridae. It causes stomatitis vesicularis, an infectious disease affecting hooved mammals (cattle, horse and pig). In humans, it can cause flu-like symptoms, swelling of the lymph nodes and neurological side-effects.

It is conceivable to design a recombinant vaccine vector against EBOV based on NDV. This would ensure an uninhibited IFN response with all its positive consequences as discussed in the present review. Whether a mammalian virus such as VSV can exert an uninhibited and similarly strong IFN response as that of NDV in other mammals, including man, remains to be investigated.

\section{Acknowledgements}

The author acknowledges financial support from the German Cancer Research Center, Heidelberg, at which the study on murine $\mathrm{KO}$ cells was performed.

\section{References}

1. Pestka S, Krause CD and Walter MR: Interferons, interferon-like cytokines, and their receptors. Immunol Rev 202: 8-32, 2004.

2. Levy DE and García-Sastre A: The virus battles: IFN induction of the antiviral state and mechanisms of viral evasion. Cytokine Growth Factor Rev 12: 143-156, 2001.

3. González-Navajas JM, Lee J, David M and Raz E: Immunomodulatory functions of type I interferons. Nat Rev Immunol 12 125-135, 2012

4. Kadowaki N, Antonenko S, Lau JY and Liu YJ: Natural interferon alpha/beta-producing cells link innate and adaptive immunity. J Exp Med 192: 219-226, 2000.

5. Taniguchi T, Mantei N, Schwarzstein M, et al: Human leukocyte and fibroblast interferons are structurally related. Nature 285 : 547-549, 1980.

6. Shuai K and Liu B: Regulation of JAK-STAT signalling in the immune system. Nat Rev Immunol 3: 900-911, 2003.

7. Barber GN: Host defense, viruses and apoptosis. Cell Death Differ 8: 113-126, 2001

8. Jarvis ED, Mirarab S, Aberer AJ, Li B, Houde P, Li C, Ho SY, Faircloth BC, Nabholz B, Howard JT, et al: Whole-genome analyses resolve early branches in the tree of life of modern birds. Science 346: 1320-1331, 2014.

9. Alexander DJ (ed): Newcastle Disease. Kluwer Academic Publishers, Boston, MA, 1988.

10. Schirrmacher V and Fournier P: Newcastle disease virus: A promising vector for viral therapy, immune therapy, and gene therapy of cancer (Review). Methods Mol Biol 542: 565-605, 2009.

11. Fournier P and Schirrmacher V: Oncolytic Newcastle Disease Virus as cutting edge between tumor and host. Biology (Basel) 2 : 936-975, 2013.

12. Hanson RP and Brandly CA: Identification of vaccine strains of Newcastle disease virus. Science 122: 156-157, 1955.

13. Mebatsion T, de Vaan LT, de Haas N, Römer-Oberdörfer A and Braber M: Identification of a mutation in editing of defective Newcastle disease virus recombinants that modulates P-gene mRNA editing and restores virus replication and pathogenicity in chicken embryos. J Virol 77: 9259-9265, 2003.

14. Huang Z, Krishnamurthy S, Panda A and Samal SK: Newcastle disease virus $\mathrm{V}$ protein is associated with viral pathogenesis and functions as an alpha interferon antagonist. J Virol 77: 8676-8685, 2003.
15. Park MS,Shaw ML, Muñoz-Jordan J,Cros JF,Nakaya T, BouvierN, Palese P, García-Sastre A and Basler CF: Newcastle disease virus (NDV)-based assay demonstrates interferon-antagonist activity for the NDV $\mathrm{V}$ protein and the Nipah virus $\mathrm{V}, \mathrm{W}$, and $\mathrm{C}$ proteins. J Virol 77: 1501-1511, 2003.

16. Park MS, García-Sastre A, Cros JF, Basler CF and Palese P: Newcastle disease virus $\mathrm{V}$ protein is a determinant of host range restriction. J Virol 77: 9522-9532, 2003.

17. Horvath CM: Weapons of STAT destruction. Interferon evasion by paramyxovirus V protein. Eur J Biochem 271: 4621-4628, 2004.

18. Hengel H, Koszinowski UH and Conzelmann KK: Viruses know it all: new insights into IFN networks. Trends Immunol 26: 396-401, 2005

19. Zaslavsky E, Hershberg U, Seto J,Pham AM, Marquez S, Duke JL, Wetmur JG, Tenoever BR, Sealfon SC and Kleinstein SH: Antiviral response dictated by choreographed cascade of transcription factors. J Immunol 184: 2908-2917, 2010.

20. Wilden H, Fournier P, Zawatzky R and Schirrmacher V: Expression of RIG-I, IRF3, IFN-beta and IRF7 determines resistance or susceptibility of cells to infection by Newcastle Disease Virus. Int J Oncol 34: 971-982, 2009.

21. Fournier P, Wilden H and Schirrmacher V: Importance of retinoic acid-inducible gene I and of receptor for type I interferon for cellular resistance to infection by Newcastle disease virus. Int J Oncol 40: 287-298, 2012.

22. Hornung V,Ellegast J,Kim S, Brzózka K, Jung A, KatoH, Poeck H, Akira S, Conzelmann KK, Schlee M, et al: 5'-Triphosphate RNA is the ligand for RIG-I. Science 314: 994-997, 2006.

23. Taniguchi $\mathrm{T}$ and Takaoka A: The interferon-alpha/beta system in antiviral responses: A multimodal machinery of gene regulation by the IRF family of transcription factors. Curr Opin Immunol 14: 111-116, 2002.

24. Wilden H, Schirrmacher V and Fournier P: Important role of interferon regulatory factor (IRF)-3 in the interferon response of mouse macrophages upon infection by Newcastle disease virus. Int J Oncol 39: 493-504, 2011.

25. Goubau D, Schlee M, Deddouche S, Pruijssers AJ, Zillinger T, Goldeck M, Schuberth C, Van der Veen AG, Fujimura T, Rehwinkel J, et al: Antiviral immunity via RIG-I-mediated recognition of RNA bearing 5'-diphosphates. Nature 514: 372-375, 2014

26. Zhang X, Li S, Luo Y, Chen Y, Cheng S, Zhang G, Hu C, Chen H and Guo A: Mycobacterium bovis and BCG induce different patterns of cytokine and chemokine production in dendritic cells and differentiation patterns in $\mathrm{CD} 4^{+} \mathrm{T}$ cells. Microbiology 159: 366-379, 2013.

27. Grandvaux N, Servant MJ, tenOever B, Sen GC, Balachandran S, Barber GN, Lin R and Hiscott J: Transcriptional profiling of interferon regulatory factor 3 target genes: direct involvement in the regulation of interferon-stimulated genes. J Virol 76: 5532-5539, 2002.

28. Sen GC and Peters GA: Viral stress-inducible genes. Adv Virus Res 70: 233-263, 2007.

29. Tailor P, Tamura T and Ozato K: IRF family proteins and type I interferon induction in dendritic cells. Cell Res 16: 134-140, 2006.

30. Ivashkiv LB and Donlin LT: Regulation of type I interferon responses. Nat Rev Immunol 14: 36-49, 2014.

31. Parks GD and Alexander-Miller MA: Paramyxovirus activation and inhibition of innate immune responses. J Mol Biol 425: 4872-4892, 2013

32. Fournier P, Arnold A, Wilden H and Schirrmacher V: Newcastle disease virus induces pro-inflammatory conditions and type I interferon for counter-acting Treg activity. Int J Oncol 40: 840-850, 2012.

33. Fournier P, Arnold A and Schirrmacher V: Polarization of human monocyte-derived dendritic cells to $\mathrm{DC} 1$ by in vitro stimulation with Newcastle Disease Virus. J BUON 14 (Suppl): S111-S122, 2009.

34. Weber F, Kochs G and Haller O: Inverse interference: how viruses fight the interferon system. Viral Immunol 17: 498-515, 2004.

35. Jenner RG and Young RA: Insights into host responses against pathogens from transcriptional profiling. Nat Rev Microbiol 3: 281-294, 2005.

36. Huang Q, Liu D, Majewski P, Schulte LC, Korn JM, Young RA, Lander ES and Hacohen N: The plasticity of dendritic cell responses to pathogens and their components. Science 294: 870-875, 2001.

37. Fiola C, Peeters B, Fournier P, Arnold A, Bucur M and Schirrmacher V: Tumor selective replication of Newcastle disease virus: Association with defects of tumor cells in antiviral defence. Int J Cancer 119: 328-338, 2006. 
38. Jarahian M, Watzl C, Fournier P, Arnold A, Djandji D, Zahedi S, Cerwenka A, Paschen A, Schirrmacher V and Momburg F: Activation of natural killer cells by newcastle disease virus hemagglutinin-neuraminidase. J Virol 83: 8108-8121, 2009.

39. Washburn B, Weigand MA, Grosse-Wilde A, Janke M, Stahl H, Rieser E, Sprick MR, Schirrmacher V and Walczak H: TNF-related apoptosis-inducing ligand mediates tumoricidal activity of human monocytes stimulated by Newcastle disease virus. J Immunol 170: 1814-1821, 2003

40. Termeer CC, Schirrmacher V, Bröcker EB and Becker JC: Newcastle disease virus infection induces B7-1/B7-2-independent T-cell costimulatory activity in human melanoma cells. Cancer Gene Ther 7: 316-323, 2000.

41. Ertel C, Millar NS, Emmerson PT, Schirrmacher V and von Hoegen P: Viral hemagglutinin augments peptide-specific cytotoxic T cell responses. Eur J Immunol 23: 2592-2596, 1993.

42. Umansky V, Shatrov VA, Lehmann V and Schirrmacher V: Induction of NO synthesis in macrophages by Newcastle disease virus is associated with activation of nuclear factor-kappa B. Int Immunol 8: 491-498, 1996.

43. Klenk HD (ed): Marburg and Ebola Viruses. In: Current Topics in Microbiol and Immunol 235. Springer, 1999.

44. Bray M and Geisbert TW: Ebola virus: The role of macrophages and dendritic cells in the pathogenesis of Ebola hemorrhagic fever. Int J Biochem Cell Biol 37: 1560-1566, 2005.

45. WHO Ebola Response Team: Ebola virus disease in West Africa. New Engl J Med 371: 1481-1495, 2014.

46. Zhang L, Wang H and Zhang YQ: Against Ebola: Type I interferon guard risk and mesenchymal stromal cell combat sepsis. J Zhejiang Univ Sci B 16: 1-9, 2015.

47. Ramanan P, Shabman RS, Brown CS, Amarasinghe GK, Basler CF and Leung DW: Filoviral immune evasion mechanisms. Viruses 3: 1634-1649, 2011.

48. Leung DW, Ginder ND, Fulton DB, Nix J, Basler CF, Honzatko RB and Amarasinghe GK: Structure of the Ebola VP35 interferon inhibitory domain. Proc Natl Acad Sci USA 106: 411-416, 2009.

49. Xu W, Edwards MR, Borek DM, Feagins AR, Mittal A, Alinger JB, Berry KN, Yen B, Hamilton J, Brett TJ, et al: Ebola virus VP24 targets a unique NLS binding site on karyopherin alpha 5 to selectively compete with nuclear import of phosphorylated STAT1. Cell Host Microbe 16: 187-200, 2014.

50. Bale S, Julien JP, Bornholdt ZA, Kimberlin CR, Halfmann P, Zandonatti MA, Kunert J,Kroon GJ,Kawaoka Y,MacRae IJ, et al: Marburg virus VP35 can both fully coat the backbone and cap the ends of dsRNA for interferon antagonism. PLoS Pathog 8: e1002916, 2012

51. Leung DW, Prins KC, Borek DM, Farahbakhsh M, Tufariello JM, Ramanan P, Nix JC, Helgeson LA, Otwinowski Z, Honzatko RB, et al: Structural basis for dsRNA recognition and interferon antagonism by Ebola VP35. Nat Struct Mol Biol 17: $165-172,2010$
52. Basler CF, Mikulasova A, Martinez-Sobrido L, Paragas J, Mühlberger E, Bray M, Klenk HD, Palese P and García-Sastre A: The Ebola virus VP35 protein inhibits activation of interferon regulatory factor 3. J Virol 77: 7945-7956, 2003.

53. Feng Z, Cerveny M, Yan Z and He B: The VP35 protein of Ebola virus inhibits the antiviral effect mediated by double-stranded RNA-dependent protein kinase PKR. J Virol 81: 182-192, 2007.

54. Hartman AL, Ling L, Nichol ST and Hibberd ML: Whole-genome expression profiling reveals that inhibition of host innate immune response pathways by Ebola virus can be reversed by a single amino acid change in the VP35 protein. J Virol 82: 5348-5358, 2008.

55. Zhang AP, Bornholdt ZA, Liu T, Abelson DM, Lee DE, Li S,Woods VL Jr and Saphire EO: The ebola virus interferon antagonist VP24 directly binds STAT1 and has a novel, pyramidal fold. PLoS Pathog 8: e1002550, 2012.

56. Kulkarni RR, Rasheed MA, Bhaumik SK, Ranjan P, Cao W, Davis C, Marisetti K, Thomas S, Gangappa S, Sambhara S, et al: Activation of the RIG-I pathway during influenza vaccination enhances the germinal center reaction, promotes $\mathrm{T}$ follicular helper cell induction, and provides a dose-sparing effect and protective immunity. J Virol 88: 13990-14001, 2014

57. Cervantes-Barragán L, Kalinke U, Züst R, König M, Reizis B, López-Macías C, Thiel V and Ludewig B: Type I IFN-mediated protection of macrophages and dendritic cells secures control of murine coronavirus infection. J Immunol 182: 1099-1106, 2009.

58. Ayllon J and García-Sastre A: The NS1 protein: A multitasking virulence factor. Curr Top Microbiol Immunol 386: 73-107, 2015.

59. Reder AT, Oger JF, Kappos L, O'Connor P and Rametta M: Short-term and long-term safety and tolerability of interferon $\beta-1 b$ in multiple sclerosis. Mult Scler Relat Disord 3: 294-302, 2014.

60. Corti D and Lanzavecchia A: Broadly neutralizing antiviral antibodies. Annu Rev Immunol 31: 705-742, 2013.

61. Corti D, Sallusto F and Lanzavecchia A: High throughput cellular screens to interrogate the human $\mathrm{T}$ and $\mathrm{B}$ cell repertoires. Curr Opin Immunol 23: 430-435, 2011.

62. Sobarzo A, Eskira Y, Herbert AS, Kuehne AI, Stonier SW, Ochayon DE, Fedida-Metula S, Balinandi S, Kislev Y, Tali N, et al: Immune memory to Sudan virus: Comparison between two separate disease outbreaks. Viruses 7: 37-51, 2015.

63. Marzi A, Engelmann F, Feldmann F, Haberthur K, Shupert WL Brining D, Scott DP, Geisbert TW, Kawaoka Y, Katze MG, et al: Antibodies are necessary for rVSV/ZEBOV-GP-mediated protection against lethal Ebola virus challenge in nonhuman primates. Proc Natl Acad Sci USA 110: 1893-1898, 2013.

64. Barber GN: VSV-tumor selective replication and protein translation. Oncogene 24: 7710-7719, 2005. 